\title{
Non-Perturbative Approach to Nearly Antiferromagnetic Fermi Liquids
}

\author{
P. Monthoux \\ Cavendish Laboratory, University of Cambridge \\ Madingley Road, Cambridge, CB3 OHE, UK
}

(October 6, 2017)

\begin{abstract}
We present a non-perturbative approach to the problem of quasiparticles coupled to spin-fluctuations. If the fully dressed spin-fluctuation propagator is used in the Feynman graph expansion of the single particle Green's function, the problem of summing all spin-fluctuation exchange Feynman graphs (i.e all the graphs without fermion loops) can be cast as a functional integral over Gaussian distributed random vector fields. The quasiparticle propagator is then obtained as the average over all field configurations of the non-interacting electron Green's function in an external field. A Monte Carlo sampling of this functional integral does not suffer from the "fermion sign problem" and offers an attractive alternative to perturbative calculations. We compare the results of our computer simulations to perturbation theory and self-consistent Eliashberg calculations.
\end{abstract}




\section{INTRODUCTION}

In previous papers (Monthoux and Pines, 1993, 1994) we described the results of extensive one-loop self-consistent calculations on the normal state and superconducting transition temperature for the optimally doped copper oxide superconductor $\mathrm{YBa}_{2} \mathrm{Cu}_{3} \mathrm{O}_{7}$. In these calculations, the effective low-energy quasiparticle-quasiparticle interaction is expressed as the exchange of dressed magnetic excitations whose spectrum is determined by fits to NMR experiments. These Eliashberg calculations demonstrated that while strong coupling effects reduce $\mathrm{T}_{\mathrm{c}}$ substantially, $\mathrm{d}_{\mathrm{x}^{2}-\mathrm{y}^{2}}$ superconductivity at high temperatures is still possible with a quasiparticle-spin-fluctuation coupling constant $\mathrm{g}^{2}$ in the range required to explain the observed normal-state resisitivity and optical conductivity.

Questions regarding the validity of the single spin-fluctuation exchange picture have been raised (Schrieffer, 1995). The role of vertex corrections in the paramagnetic phase has been examined in detail by Chubukov et al. (1997) and the author (1997). For a large Fermi surface consistent with ARPES experiments on $\mathrm{YBa}_{2} \mathrm{Cu}_{3} \mathrm{O}_{7}$, vertex corrections enhance the effective spin-fluctuation interaction as the antiferromagnetic correlation length increases. The effect is strongly anisotropic and most important for quasiparticles at the hot spots (Hlubina and Rice, 1997) for which the Midgal argument does not carry through. A quasiparticle near the hot spot can emit or absorb any number of spin-fluctuations at

the antiferromagnetic wavevector $\overrightarrow{\mathrm{Q}}=(\pi, \pi)$ where the interaction is strong while staying close to the Fermi surface. One would thus expect that as the coupling gets stronger or as the correlation length increases, two, three, etc.. spin-fluctuation exchange graphs become important and perturbation theory breaks down. Non-perturbative methods are thus needed to study the behaviour of hot quasiparticles for long correlation lengths. In the case where the charactersitic spin-fluctuation frequency $\omega_{\mathrm{SF}}$ is much smaller than $2 \pi \mathrm{T}$, only the zero Matsubara frequency of the spin-fluctuation propagator is relevant and a solution to the problem in this quasi-static limit has been proposed by Schmalian et al (1998).

In this paper, the calculation of the single particle Green's function for an arbitrary 
number of spin-fluctuation exchanges is formulated as the average of the non-interacting electron Green's function in an external magnetic field whose probability distribution is Gaussian. Upon expanding the Green's function in powers of the magnetic field and doing the averages with Wick's theorem, one recovers the standard time-ordered perturbation series for the quasiparticle propagator. However, sampling the functional integral by the Monte Carlo method combined with a numerical calculation of the Green's function for each external field configuration provides an attractive and non-perturbative method of solution when straightforward perturbation theory is expected to break down.

The low-energy effective action for the planar quasiparticles is given by

$$
\begin{aligned}
\mathrm{S}_{\text {eff }}= & \sum_{\overrightarrow{\mathrm{p}}, \alpha} \int_{0}^{\beta} \mathrm{d} \tau \psi_{\overrightarrow{\mathrm{p}}, \alpha}^{\dagger}(\tau)\left(\partial_{\tau}+\epsilon_{\overrightarrow{\mathrm{p}}}-\mu\right) \psi_{\overrightarrow{\mathrm{p}}, \alpha}(\tau) \\
& -\frac{\mathrm{g}^{2}}{6} \sum_{\overrightarrow{\mathrm{q}}} \int_{0}^{\beta} \mathrm{d} \tau \int_{0}^{\beta} \mathrm{d} \tau^{\prime} \chi\left(\overrightarrow{\mathrm{q}}, \tau-\tau^{\prime}\right) \overrightarrow{\mathrm{s}}(\overrightarrow{\mathrm{q}}, \tau) \cdot \overrightarrow{\mathrm{s}}\left(-\overrightarrow{\mathrm{q}}, \tau^{\prime}\right)
\end{aligned}
$$

The spin density $\overrightarrow{\mathrm{s}}(\overrightarrow{\mathrm{q}}, \tau)$ is given by

$$
\overrightarrow{\mathrm{s}}(\overrightarrow{\mathrm{q}}, \tau) \equiv \sum_{\overrightarrow{\mathrm{p}}, \alpha, \gamma} \psi_{\overrightarrow{\mathrm{p}}+\overrightarrow{\mathrm{q}}, \alpha}^{\dagger}(\tau) \vec{\sigma}_{\alpha, \gamma} \psi_{\overrightarrow{\mathrm{p}}, \gamma}(\tau)
$$

where $\vec{\sigma}$ denotes the three Pauli matrices. The quasiparticle dispersion relation is

$$
\epsilon_{\overrightarrow{\mathrm{p}}}=-2 \mathrm{t}\left(\cos \left(\mathrm{p}_{\mathrm{x}}\right)+\cos \left(\mathrm{p}_{\mathrm{y}}\right)\right)-4 \mathrm{t}^{\prime} \cos \left(\mathrm{p}_{\mathrm{x}}\right) \cos \left(\mathrm{p}_{\mathrm{y}}\right)
$$

with hopping matrix elements $\mathrm{t}=0.25 \mathrm{eV}$ and t'=-0.45t, as suggested by $\mathrm{Si}$ et al. (1993) from fits to band theory and ARPES experiments. We use units in which the lattice constant is unity. $\mu$ denotes the chemical potential, $\beta$ the inverse temperature, $\mathrm{g}^{2}$ the coupling constant and $\psi_{\overrightarrow{\mathrm{p}}, \sigma}^{\dagger}$ and $\psi_{\overrightarrow{\mathrm{p}}, \sigma}$ are Grassmann variables. A coupling constant $\mathrm{g}^{2}=0.41 \mathrm{eV}^{2}$ gives an

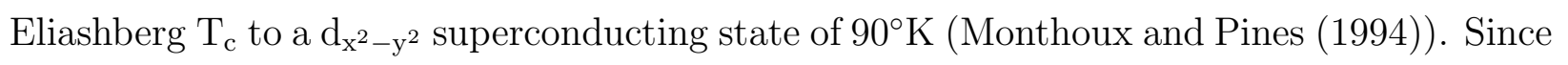
the interaction $\chi(\overrightarrow{\mathrm{q}}, \tau)$ is determined from fits to normal state experiments, it is fully dressed. Therefore, in a Feynman graph expansion, the diagrams corresponding to a renormalization of the spin-fluctuation propagator $\chi(\overrightarrow{\mathrm{q}}, \tau)$ should not be included. On the real frequency axis, $\chi(\overrightarrow{\mathrm{q}}, \omega)$ is assumed to take the phenomenological form introduced by Millis et al.(1990) 


$$
\chi(\overrightarrow{\mathrm{q}}, \omega)=\frac{\chi_{\overrightarrow{\mathrm{Q}}}}{1+\xi^{2}(\overrightarrow{\mathrm{q}}-\overrightarrow{\mathrm{Q}})^{2}-\mathrm{i} \frac{\omega}{\omega_{\mathrm{SF}}}}
$$

Here $\chi_{\vec{Q}}$ is the static spin susceptibility at wavevector $\vec{Q}=(\pi, \pi)$ and $\xi$ is a temperature dependent antiferromagnetic correlation length. The frequency $\omega_{\text {SF }}$ defines the charactersitic energy of the low-frequency magnetic response. The quantities $\chi_{\overrightarrow{\mathrm{Q}}}$ and $\omega_{\mathrm{SF}}$ may be expressed in terms of the experimentally measured long-wavelength spin susceptibility $\chi_{0}$ and a shortdistance characteristic spin-fluctuation energy $\Gamma_{\mathrm{AF}}$ by introducing a scale factor $\beta$ (not to be confused with the inverse temperature)

$$
\begin{aligned}
& \chi_{\overrightarrow{\mathrm{Q}}}=\chi_{0} \sqrt{\beta} \xi^{2} \\
& \omega_{\mathrm{SF}}=\frac{\Gamma_{\mathrm{AF}}}{\sqrt{\beta} \pi \xi^{2}}
\end{aligned}
$$

We adopt the same values of these parameters as in Monthoux and Pines (1994), namely $\Gamma_{\mathrm{AF}}=1.3 \mathrm{eV}, \chi_{0}=2.6$ states $/ \mathrm{eV}, \beta=32$. The temperature dependence of the charactersitic spin-fluctuation energy $\omega_{\mathrm{SF}}$ is taken as

$$
\omega_{\mathrm{SF}}(\mathrm{T})=\left(9.5+4.75\left[\mathrm{~T}\left({ }^{\circ} \mathrm{K}\right) / 100\right]\right) \mathrm{meV}
$$

from which the temperature-dependent antiferromagnetic correlation length can be obtained using Eq. (6). The numerical values of these parameters for $\mathrm{YBa}_{2} \mathrm{Cu}_{3} \mathrm{O}_{7}$ are $\chi_{\overrightarrow{\mathrm{Q}}}\left(\mathrm{T}_{\mathrm{c}}\right) \approx$ 75 states $/ \mathrm{eV}, \xi\left(\mathrm{T}_{\mathrm{c}}\right) \approx 2.3$ and $\omega_{\mathrm{SF}}\left(\mathrm{T}_{\mathrm{c}}\right) \approx 14 \mathrm{meV}$.

The spin-fluctuation propagator on the imaginary axis, $\chi\left(\overrightarrow{\mathrm{q}}, \mathrm{i} \nu_{\mathrm{n}}\right)$ is related to the imaginary part of the response function $\operatorname{Im} \chi(\overrightarrow{\mathrm{q}}, \omega)$, Eq. (4), via the spectral representation

$$
\chi\left(\overrightarrow{\mathrm{q}}, \mathrm{i} \nu_{\mathrm{n}}\right)=-\int_{-\infty}^{+\infty} \frac{\mathrm{d} \omega}{\pi} \frac{\operatorname{Im} \chi(\overrightarrow{\mathrm{q}}, \omega)}{\mathrm{i} \nu_{\mathrm{n}}-\omega}
$$

To get $\chi\left(\overrightarrow{\mathrm{q}}, \mathrm{i} \nu_{\mathrm{n}}\right)$ to decay as $1 / \nu_{\mathrm{n}}^{2}$ as $\nu_{\mathrm{n}} \rightarrow \infty$, as it should, we introduce a cutoff $\omega_{0}$ and take $\operatorname{Im} \chi(\overrightarrow{\mathrm{q}}, \omega)=0$ for $\omega \geq \omega_{0}$. In the following, we adopt the value $\omega_{0}=0.4 \mathrm{eV}$. 


\section{FUNCTIONAL INTEGRAL FORMULATION}

In this section, we formulate the problem of calculating the single particle Green's function for an arbitrary number of spin-fluctuation exchanges as a functional integral that is suitable for computer simulation.

Let us first introduce some notation. In the following, we denote by $\mathrm{x}$ the position and imaginary time coordinate, $\mathrm{x} \equiv(\overrightarrow{\mathrm{x}}, \tau)$ and similarly, let $\mathrm{q}$ stand for the wavevector and Matsubara frequency 3 -vector $\mathrm{q} \equiv\left(\overrightarrow{\mathrm{q}}, \mathrm{i} \nu_{\mathrm{n}}\right)$. Consider a real vector field $\overrightarrow{\mathrm{M}}(\mathrm{x})$, such that

$$
\overrightarrow{\mathrm{M}}(-\mathrm{q})=\overrightarrow{\mathrm{M}}^{*}(\mathrm{q})
$$

and introduce the average $\langle\ldots\rangle$ over the Gaussian probability distribution of the fields $\overrightarrow{\mathrm{M}}(\mathrm{q})$ :

$$
<\ldots>\equiv \frac{1}{\mathrm{Z}} \iint \mathrm{D} \overrightarrow{\mathrm{M}} \ldots \exp \left(-\sum_{\mathrm{q}} \frac{\overrightarrow{\mathrm{M}}(\mathrm{q}) \cdot \overrightarrow{\mathrm{M}}(-\mathrm{q})}{2 \alpha(\mathrm{q})}\right)
$$

where $\alpha(\mathrm{q})$ is real and the normalization factor $\mathbf{Z}$ is defined as

$$
\mathrm{Z}=\iint \mathrm{D} \overrightarrow{\mathrm{M}} \exp \left(-\sum_{\mathrm{q}} \frac{\overrightarrow{\mathrm{M}}(\mathrm{q}) \cdot \overrightarrow{\mathrm{M}}(-\mathrm{q})}{2 \alpha(\mathrm{q})}\right)
$$

The integrals DM are carried out over all of the independent components of the vector field $\vec{M}(\mathrm{q})$. The two-point correlation function of the field $\overrightarrow{\mathrm{M}}(\mathrm{q})$ is straightforward to calculate and is given by

$$
<\mathrm{M}_{\mathrm{i}}(\mathrm{q}) \mathrm{M}_{\mathrm{j}}(\mathrm{k})>=\delta_{\mathrm{i}, \mathrm{j}} \delta_{\mathrm{k},-\mathrm{q}} \times\left\{\begin{array}{c}
2 \alpha(\mathrm{q}) \text { if } \mathrm{M}(\mathrm{q}) \text { is complex } \\
\alpha(\mathrm{q}) \text { if } \mathrm{M}(\mathrm{q}) \text { is real }
\end{array}\right.
$$

Since the integrals over $\vec{M}(q)$ are Gaussian, the average of an odd number of fields vanishes and the higher order correlations can be obtained from the two-point correlation function by Wick's theorem. For instance the four-point correlation function is given by:

$$
\begin{aligned}
<\mathrm{M}_{\mathrm{i}}(\mathrm{q}) \mathrm{M}_{\mathrm{j}}(\mathrm{k}) \mathrm{M}_{\mathrm{n}}(\mathrm{p}) \mathrm{M}_{\mathrm{m}}(\mathrm{l})>= & <\mathrm{M}_{\mathrm{i}}(\mathrm{q}) \mathrm{M}_{\mathrm{j}}(\mathrm{k})><\mathrm{M}_{\mathrm{n}}(\mathrm{p}) \mathrm{M}_{\mathrm{m}}(\mathrm{l})> \\
& +<\mathrm{M}_{\mathrm{i}}(\mathrm{q}) \mathrm{M}_{\mathrm{n}}(\mathrm{p})><\mathrm{M}_{\mathrm{j}}(\mathrm{k}) \mathrm{M}_{\mathrm{m}}(\mathrm{l})> \\
& +<\mathrm{M}_{\mathrm{i}}(\mathrm{q}) \mathrm{M}_{\mathrm{m}}(\mathrm{l})><\mathrm{M}_{\mathrm{j}}(\mathrm{k}) \mathrm{M}_{\mathrm{n}}(\mathrm{p})>
\end{aligned}
$$


Now we choose $\alpha(q)$ such that

$$
<\mathrm{M}_{\mathrm{i}}(\mathrm{q}) \mathrm{M}_{\mathrm{i}}(-\mathrm{q})>=\frac{\mathrm{g}^{2}}{3} \frac{\mathrm{T}}{\mathrm{N}} \chi(\mathrm{q})
$$

where $\mathrm{N}$ is the number of momenta in the Brillouin Zone, $\mathrm{T}$ the temperature and let

$$
\tilde{\mathrm{G}} \equiv<\tilde{\mathrm{G}}_{0}\left[1-\tilde{\mathrm{M}} \tilde{\mathrm{G}}_{0}\right]^{-1}>
$$

where the matrices $\tilde{\mathrm{G}}_{0}$ and $\tilde{\mathrm{M}}$ in 3 -momentum and spin space are defined as

$$
\begin{aligned}
& {\left[\tilde{\mathrm{G}}_{0}\right]_{(\mathrm{q}, \alpha)\left(\mathrm{q}^{\prime}, \beta\right)}=\mathrm{G}_{0}(\mathrm{q}) \delta_{(\mathrm{q}, \alpha),\left(\mathrm{q}^{\prime}, \beta\right)}} \\
& {[\tilde{\mathrm{M}}]_{(\mathrm{q}, \alpha)\left(\mathrm{q}^{\prime}, \beta\right)}=\vec{\sigma}_{\alpha, \beta} \cdot \overrightarrow{\mathrm{M}}\left(\mathrm{q}-\mathrm{q}^{\prime}\right)}
\end{aligned}
$$

where $\vec{\sigma}$ are the three Pauli matrices and $\alpha, \beta$ spin indices. Upon expanding and doing the averages over the random fields $\vec{M}$, one generates the following perturbation expansion:

$$
\begin{aligned}
\tilde{\mathrm{G}} \equiv & <\tilde{\mathrm{G}}_{0}\left[1-\tilde{\mathrm{M}} \tilde{\mathrm{G}}_{0}\right]^{-1}> \\
& =\tilde{\mathrm{G}}_{0}+<\tilde{\mathrm{G}}_{0} \tilde{\mathrm{M}} \tilde{\mathrm{G}}_{0} \tilde{\mathrm{M}} \tilde{\mathrm{G}}_{0}> \\
& +<\tilde{\mathrm{G}}_{0} \tilde{\mathrm{M}} \tilde{\mathrm{G}}_{0} \tilde{\mathrm{M}} \tilde{\mathrm{G}}_{0} \tilde{\mathrm{M}} \tilde{\mathrm{G}}_{0} \tilde{\mathrm{M}} \tilde{\mathrm{G}}_{0}>+\ldots
\end{aligned}
$$

The Feynman graph expansion is shown in fig.(1). One can easily convince oneself that the above formulation of the problem amounts to summing all spin-fluctuation exchange graphs. Note that no fermion loops are included. The second order dressing of the spin-fluctuation propagator and higher order RPA-like diagrams can be absorbed in the definition of the magnetic response function $\chi(q)$. Higher order fermion loops, such as the fourth order term with four spin-fluctuation lines (non-linear magnetic response), etc.. are not included in the above formulation of the problem. These are the terms that give rise to the "fermion sign problem" in determinant Monte Carlo simulations. Some of these diagrams are shown in fig.(2). The quartic term should in principle be introduced in the probability distribution of the vector field $\vec{M}(q)$ when the coefficient $\chi^{-1}(q)$ of the quadratic term becomes very small, i.e. near the phase transition. In this paper, however, we shall only consider a Gaussian distribution of the fields $\overrightarrow{\mathrm{M}}(\mathrm{q})$. 
The above formulation immediately suggests a non-perturbative approach to the problem: instead of expanding the matrix inverse and doing the integrals analytically, one can sample the functional integral by the Monte Carlo method and numerically invert the Green's function matrix in Eq. (15). The columns of the inverse are calculated by solving the appropriate linear system of equations by means of a preconditioned conjugate gradient algorithm. Since the matrix is not hermitian positive definite, there is no guaranteed convergence for the conjugate gradient method. In order to make the algorithm more robust, especially as the coupling gets strong, we combine the conjugate gradient with a continuation scheme: we invert the matrix $1-\lambda \tilde{M} \tilde{G}_{0}$ and gradually switch on the parameter $\lambda$ from zero to one. The diagonal of the matrix is used as a preconditioner.

Since upon doing the averages over all of the field configurations one restores translation invariance and other symmetries, the number of linear systems of equations that one must solve is much lower than the order of the matrix.

We have checked that the random fields $\vec{M}$ were sampled properly by calculating the two-point correlation function and comparing to the expected answer Eq. (12). A further check was to calculate the second order term in the expansion Eq. (18) by Monte Carlo sampling and compare with the computation of the appropriate Feynman diagram. The results agreed within the (small) statistical uncertainty. The conjugate gradient matrix inversion was tested on a small system ( 4 by 4 lattice) at high temperature where it could be compared to the result of Gaussian elimination with partial pivoting.

\section{RESULTS}

We shall present results for a doping $\mathrm{n}=0.8$ at a temperature $\mathrm{T}=200^{\circ} \mathrm{K}$ for a couple of coupling constants $\mathrm{g}^{2}$ and compare the results of the computer simulations to lowest order perturbation theory (i.e. one spin-fluctuation exchange Feynman graph with bare Green's function) and self-consistent one-loop calculations (i.e single spin-fluctuation exchange Feyn-

man diagram with dressed Green's function). The computations were carried out on a $16 \times 16$ 
lattice with 40 fermion and 21 boson Matsubara frequencies. With such parameters, the Green's function, Eq. (15) is obtained by inverting the 20480 by 20480 matrix 1 - $\tilde{M} \tilde{G}_{0}$ for each configuration of the random vector fields $\overrightarrow{\mathrm{M}}(\mathrm{q})$.

The Eliahsberg renormalization factor $\mathrm{Z}(\overrightarrow{\mathrm{p}}, \mathrm{i} \pi \mathrm{T})$ for a coupling constant $\mathrm{g}^{2}=0.1 \mathrm{eV}^{2}$ is shown in fig.(3). The coupling is intermediate, since there is a difference between the simple first-order perturbation theoretic calculation and the self-consistent one-loop result. Note that if one tries to calculate the Green's function, Eq. (15) for each configuration of the random field $\vec{M}$ by expanding the denominator in powers of $\vec{M}$ (using the geometric series), one finds that the expansion diverges for most field configurations. We have independently checked that the spectral radius of the matrix $\tilde{M} \tilde{G}_{0}$ is larger than one, but none of its eigenvalues are equal to one, so the inverse in Eq. (15) is well defined. In this coupling constant regime, the self-consistent calculation agrees quite well with the results of the Monte Carlo calculation. As expected, it is near the hot spots that the first-order perturbation theory and self-consistent calculations deviate from the Monte Carlo results. Away from the hot spots, the effective coupling is weak (since there is no appreciable difference between the first-order and self-consistent calculations) and perturbation theory works satifactorily. Near the hot spots, the Monte Carlo simulation yields a renormalization factor that is slightly reduced relative to the self-consistent Eliashberg result. This must be attributed to third and higher order processes, since the lowest order vertex correction enhances the effect of the interaction for quasiparticles near the hot spots (Chubukov et. al (1997), Monthoux (1997)).

The Eliahsberg renormalization factor $\mathrm{Z}(\overrightarrow{\mathrm{p}}, \mathrm{i} \pi \mathrm{T})$ for a coupling constant $\mathrm{g}^{2}=0.4 \mathrm{eV}^{2}$ is shown in fig.(4). While near cold spots the results of both the first-order perturbation theoretic and the self-consistent calculations agree with the Monte Carlo data and each other, one notes that near the hot spots the perturbation theoretic calculation does a much better job than the Eliashberg self-consistent one. The vertex corrections must thus "undress" the Eliashberg Green's function for this value of the coupling constant. Since the results of the simulations near the hot spots yield a Z some $20 \%$ larger than the Eliashberg calculation, one 
can conclude that the higher order vertex corrections enhance the spin-fluctuation interaction around the antiferromagnetic wavevector when $\mathrm{g}^{2}=0.4 \mathrm{eV}^{2}$. We have just begun the exploration of the parameter space and it will be interesting to see what happens as all of the parameters (coupling constant, antiferromagnetic correlation length and characteristic spin-fluctuation frequency) are varied.

\section{OUTLOOK}

We have presented a non-perturbative method for studying the problem of quasiparticles coupled to spin-fluctuations and compared our results to first-order perturbation theory and one-loop self-consistent calculations. We have shown some of the limitations of both these approaches. We shall study the behaviour of the hot quasiparticles as the antiferromagnetic length increases and shall look for the pseudo-gap phenomenon seen in ARPES experiments (Loeser et al.,1996), (Ding et al.,1996). As one approaches the antiferromagnetic transition, it may not be appropriate to consider only Gaussian terms in the action for the spinfluctuations. The method allows the introduction of quartic terms in the action of the random magnetic field. The study of the effect of such terms on the normal state properties of the system would be of great interest. The present formalism can in principle be extended to study other Green's functions. We are presently working on such extensions.

\section{ACKNOWLEGEMENTS}

This work was supported in part by EPSRC. I would like to thank J. Cooper, J. Loram, G. Lonzarich, C. Nex, D. Pines, D. Scalapino, J. Schmalian for helpful discussions on these and related topics. 


\section{REFERENCES}

A.V. Chubukov, P. Monthoux and D.R. Morr, Phys. Rev. B 567789 (1997).

H. Ding et al., Nature 382, 51 (1996).

R. Hlubina and T.M. Rice, Phys. Rev. B 51, 9253 (1995), Phys. Rev. B 52, 13042 (1995).

D. Loeser et al., Science 273, 325 (1996).

A.J. Millis, H. Monien and D. Pines, Phys. Rev. B 42, 167 (1990).

P. Monthoux and D. Pines, Phys. Rev. B 47, 6069 (1993).

P. Monthoux and D. Pines, Phys. Rev. B 48, 4261 (1994).

P. Monthoux, Phys. Rev. B 55, 15261 (1997).

J. Schmalian, D. Pines and B. Stojkovic, to appear in Phys. Rev. Lett. and unpublished (1998).

J. R. Schrieffer, J. Low. Temp. Phys. 99, 397 (1995).

Q. Si, Y. Zha, K. Levin, and J.P. Lu, Phys. Rev. B 47, 9055 (1993). 


\section{FIGURES}

FIG. 1. Spin-fluctuation exchange Feynman graph expansion for the quasiparticle Green's function (heavy line) obtained by expanding Eq. (15) in powers of the external field $\overrightarrow{\mathrm{M}}$.

FIG. 2. Some of the diagrams not included in the expansion. The first two RPA-like graphs amount to a renormalization of the spin-fluctuation propagator and are already accounted for by our use of the experimental $\chi(\overrightarrow{\mathrm{q}}, \omega)$. The last two diagrams amount to including $(\overrightarrow{\mathrm{M}})^{4}$ and $(\overrightarrow{\mathrm{M}})^{6}$ terms in action for the external field $\vec{M}$ and are not included in this work.

FIG. 3. Eliashberg renormalization factor $\mathrm{Z}\left(\overrightarrow{\mathrm{p}}, \mathrm{i} \omega_{\mathrm{n}}\right)=1-\frac{1}{\omega_{\mathrm{n}}} \operatorname{Im} \Sigma\left(\overrightarrow{\mathrm{p}}, \mathrm{i} \omega_{\mathrm{n}}\right)$ at $\mathrm{T}=200^{\circ} \mathrm{K}$ as a function of momentum $\vec{p}$ for the lowest Matsubara frequency $\omega_{n}=\pi \mathrm{T}$. The coupling constant is $\mathrm{g}^{2}=0.1 \mathrm{eV}^{2}$

FIG. 4. Eliashberg renormalization factor $\mathrm{Z}\left(\overrightarrow{\mathrm{p}}, \mathrm{i} \omega_{\mathrm{n}}\right)=1-\frac{1}{\omega_{\mathrm{n}}} \operatorname{Im} \Sigma\left(\overrightarrow{\mathrm{p}}, \mathrm{i} \omega_{\mathrm{n}}\right)$ at $\mathrm{T}=200^{\circ} \mathrm{K}$ as a function of momentum $\vec{p}$ for the lowest Matsubara frequency $\omega_{\mathrm{n}}=\pi \mathrm{T}$. The coupling constant is $\mathrm{g}^{2}=0.4 \mathrm{eV}^{2}$. 


$$
\begin{aligned}
& \longrightarrow==\longrightarrow+ \\
& \text { 56. }
\end{aligned}
$$

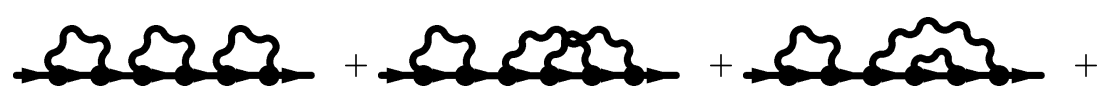

$$
\begin{aligned}
& \text { ल235 }
\end{aligned}
$$

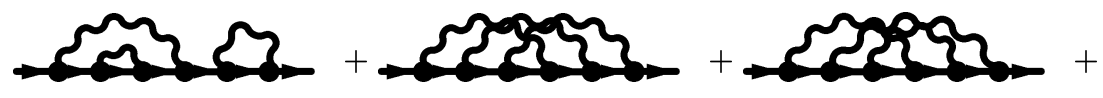

$$
\begin{aligned}
& \text { - } \\
& \text { 经政 }
\end{aligned}
$$

Fig.1 




momom
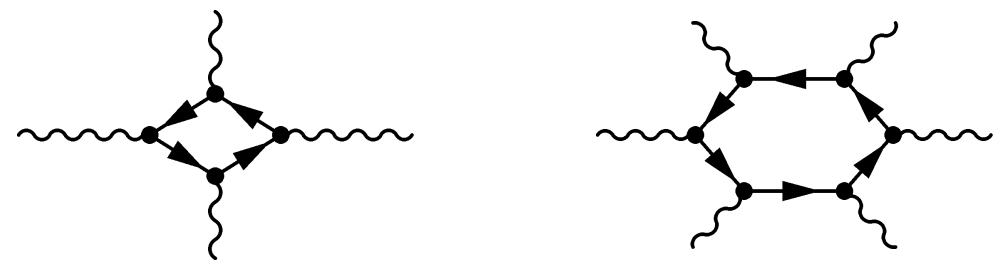

Fig.2 


\section{Eliashberg Renormalization Factor}

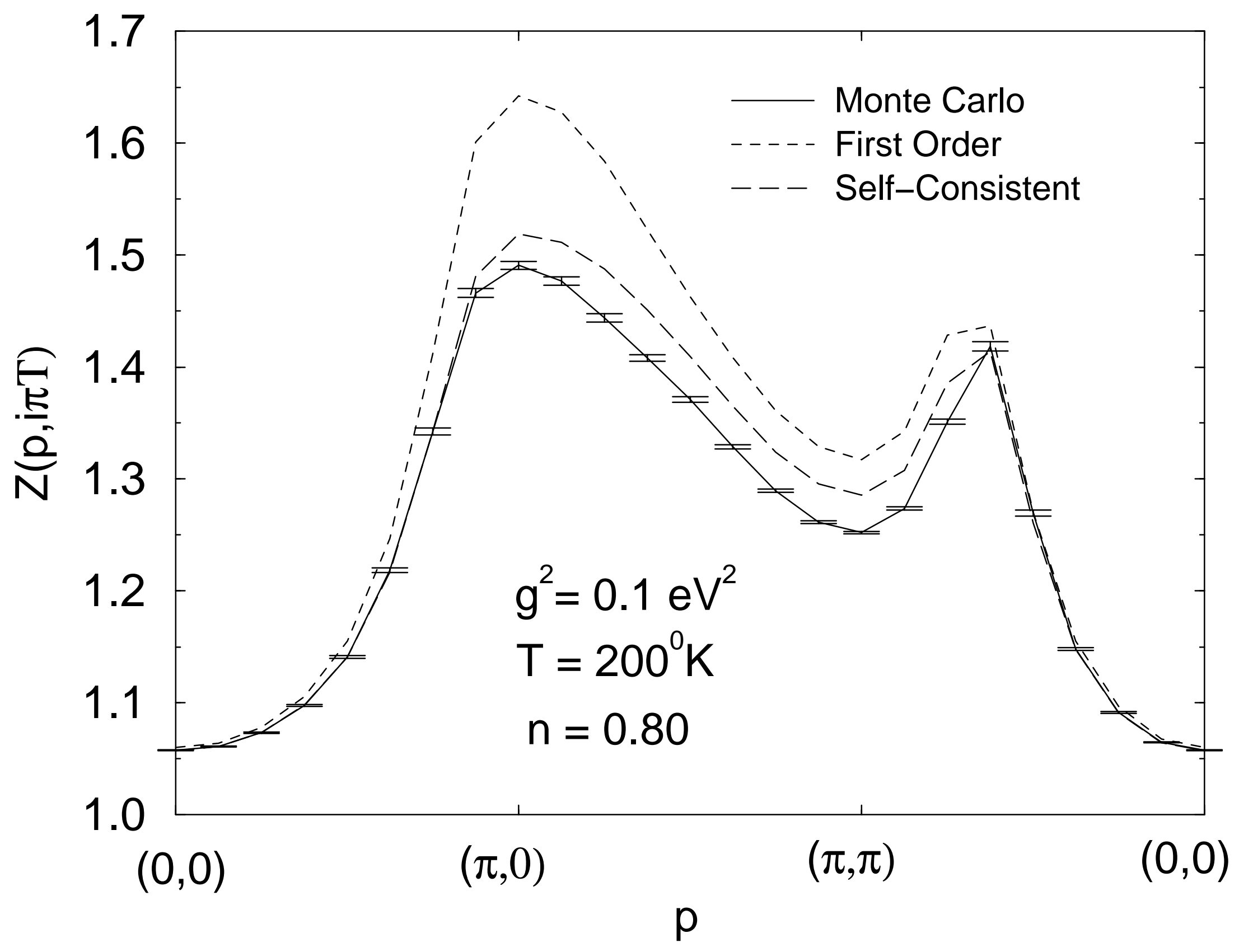


Eliashberg Renormalization Factor

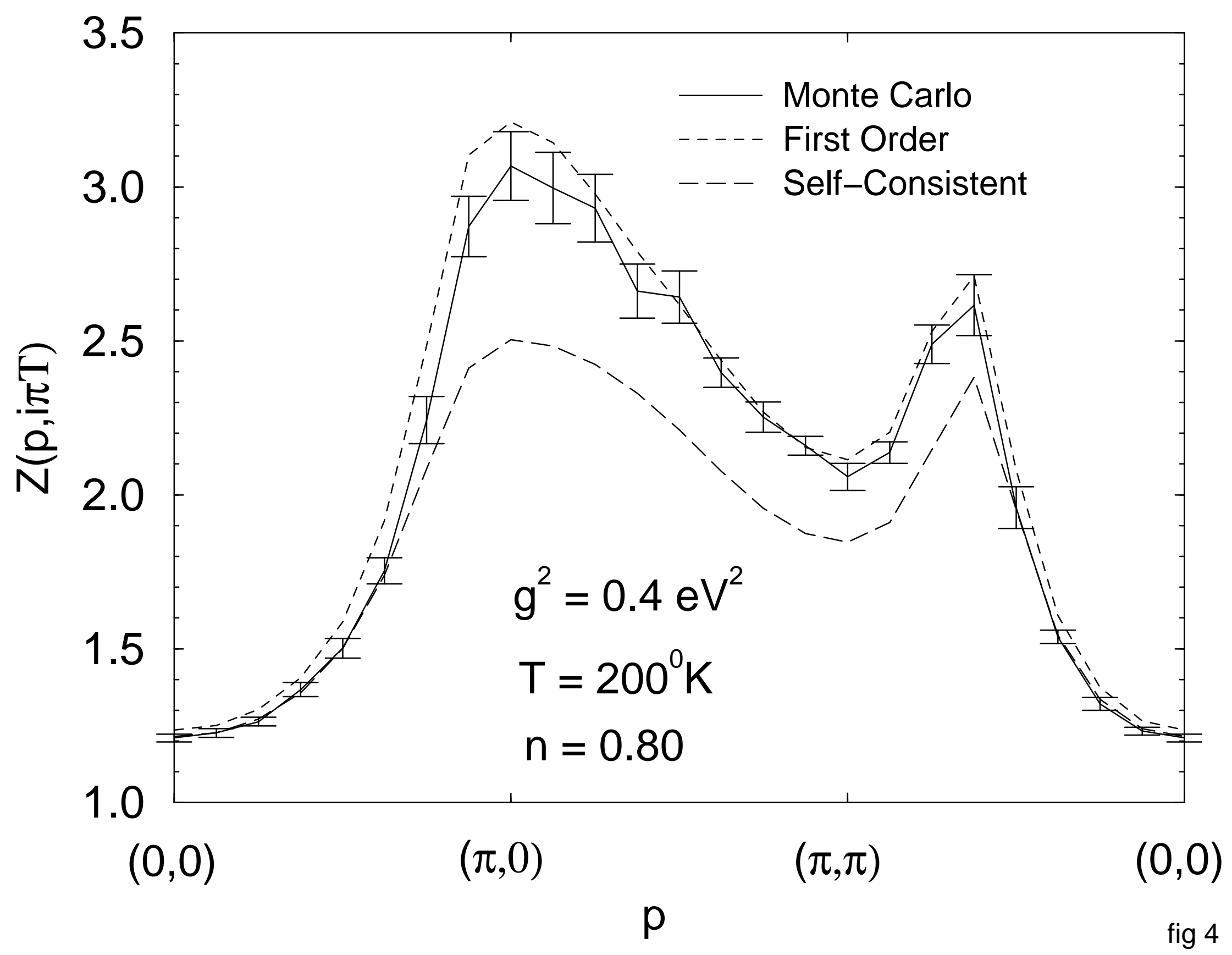

